\title{
Performance of Passive and Active Sonars in the Philippine Sea
}

\author{
Arthur B. Baggeroer \\ Massachusetts Institute of Technology \\ Departments of Mechanical and Ocean Engineering \\ Cambridge, MA 02139 \\ phone: (617) 253-4336 fax: (617) 253-2350 email: abb@ boreas.mit.edu \\ Award Number: N00014-07-1-0326 \\ http://www.onr.navy.mil/sci_tech/32/reports/annual/
}

\section{LONG-TERM GOALS}

We have the long term goals of understanding several aspects of passive and active sonar performance in the Philippine Sea when there is a robust environmental characterization of the sea floor and water column plus accurate source/receiver positions. This can lead to better signal processing where the structure and coherence can be exploited.

\section{OBJECTIVES}

The performance of the beamforming of a sonar can be separated into the gain against noise, or $N G$ and the signal gain degradation, or $S G D$. These two quantities are well understood for horizontal line arrays or arrays without much vertical extent; however, the same cannot be said when using large vertical arrays. The vertical noise structure remains and ongoing effort for VLA's Here we are concerned with sources of the SGD. Past efforts in ocean acoustics concerning the vertical structure were termed matched field processing (MFP) or coherent multipath recombination (CMR). (1) Both of these approached have not fulfilled their potential for a number of reasons including unrealistic assumptions about the coherence of the vertical field and especially the multipath and/or mode coherence.

The Philippine Sea experiments deployed two large vertical arrays, or DVLA's (Deep Vertical Line Arrays). The first experiment in 2009 had two sections at the upper and lower turning points while the second in 2010 had 140 sensors which spanned the 5600 meter water column. (4) The objective here is to determine use these data to address these coherence issues. The first issue is assess the impact of an internal wave field on the randomizing of the multipaths. This issue is controversial. A signal from a point source starts as a fully coherent signal field before any random medial effects can accumulate. The issue then is at what range does the media lead to uncorrelated multipath which is the favorite model for many in acoustical oceanography. Nevertheless, an uncorrelated vertical field essentially dooms MFP or CMR processing. If the field is coherent for a few convergence zones, this is consequential to potential of most low frequency sonar systems with vertical apertures. 


\section{Report Documentation Page}

Form Approved

OMB No. 0704-0188

Public reporting burden for the collection of information is estimated to average 1 hour per response, including the time for reviewing instructions, searching existing data sources, gathering and maintaining the data needed, and completing and reviewing the collection of information. Send comments regarding this burden estimate or any other aspect of this collection of information,

including suggestions for reducing this burden, to Washington Headquarters Services, Directorate for Information Operations and Reports, 1215 Jefferson Davis Highway, Suite 1204, Arlington

VA 22202-4302. Respondents should be aware that notwithstanding any other provision of law, no person shall be subject to a penalty for failing to comply with a collection of information if it

does not display a currently valid OMB control number.

1. REPORT DATE

30 SEP 2014

4. TITLE AND SUBTITLE

Performance of Passive and Active Sonars in the Philippine Sea

6. $\operatorname{AUTHOR}(\mathrm{S})$

7. PERFORMING ORGANIZATION NAME(S) AND ADDRESS(ES)

Massachusetts Institute of Technology,Department of Mechanical and Ocean Engineering,Cambridge,MA,02139

9. SPONSORING/MONITORING AGENCY NAME(S) AND ADDRESS(ES)
3. DATES COVERED

00-00-2014 to 00-00-2014

5a. CONTRACT NUMBER

5b. GRANT NUMBER

5c. PROGRAM ELEMENT NUMBER

5d. PROJECT NUMBER

5e. TASK NUMBER

5f. WORK UNIT NUMBER

8. PERFORMING ORGANIZATION

REPORT NUMBER

10. SPONSOR/MONITOR'S ACRONYM(S)

11. SPONSOR/MONITOR'S REPORT

NUMBER(S)

12. DISTRIBUTION/AVAILABILITY STATEMENT

Approved for public release; distribution unlimited

13. SUPPLEMENTARY NOTES

14. ABSTRACT

15. SUBJECT TERMS

16. SECURITY CLASSIFICATION OF:

a. REPORT

unclassified

b. ABSTRACT

c. THIS PAGE

unclassified
17. LIMITATION OF ABSTRACT

Same as

Report (SAR)
18. NUMBER 19a. NAME OF

OF PAGES RESPONSIBLE PERSON

5 


\section{APPROACH}

The DVLA sections for the first experiment were close enough for beamforming in the vertical; however, those for the second had 40 meter separation which leads to an aliasing angle of approximately 8 degrees. Because of all the calibrations needed to interpret the data correctly we have use simulations methods to guide our observations. This has been done by using an internal wave code implementing the Garrett- Munk model for randomizing the media and then propagating the RAM wide angle parabolic equation code through these media. ${ }^{1}$

The approach is to simulate the signals observed on a VLA generated by an axial source. The VLA receiver model has a $1 / 2$ wavelength spacing at $250 \mathrm{~Hz}$, which is the center frequency for many of the Philippine Sea transmissions and extends throughout the watercolumn to the ocean bottom. ${ }^{2}$ We then consider a sequence of subapertures with sixty four sensors which leads to each one have an approximately two degree resolution. Figure 1 illustrates the array geometry and a sample PE solution. The first step is first to identify the path structure at each range. For this apply both conventional and
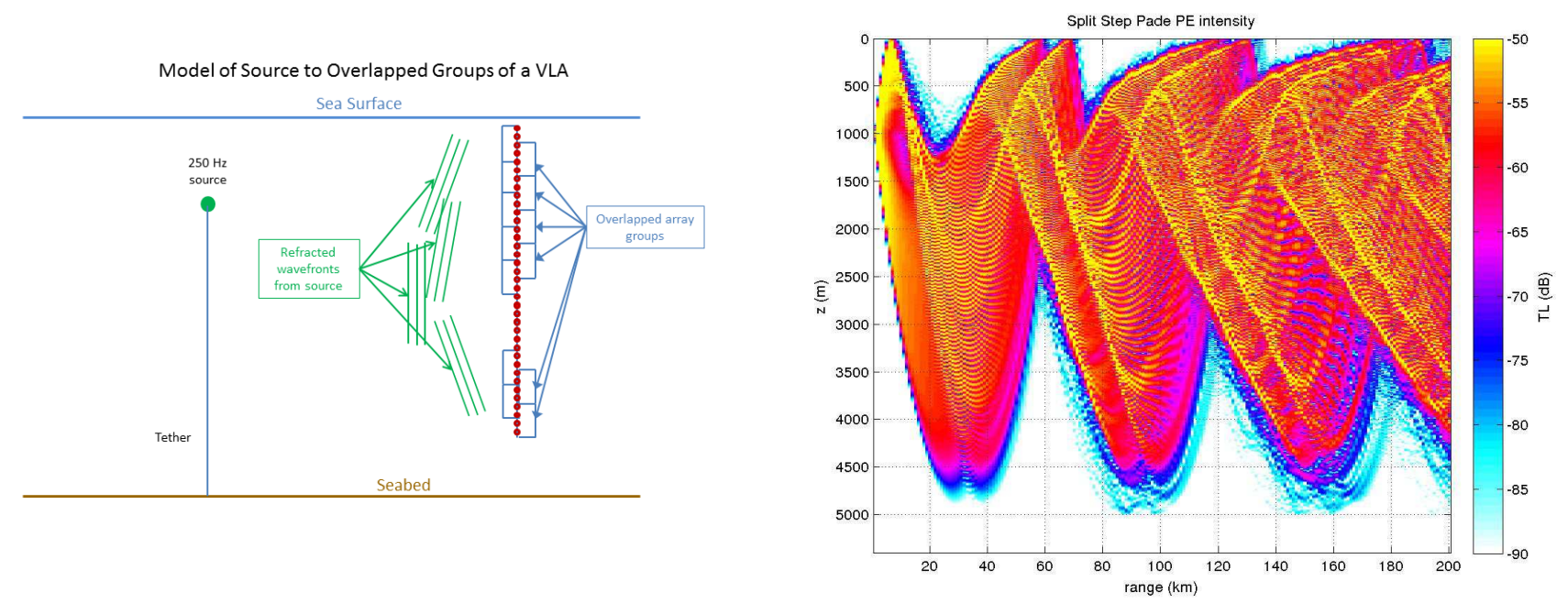

\section{Figure 1: Left: Concept of overlapped apertures on a VLA. Right: Example of RAM solution w/ Garrett-Munk perturbation}

adaptive array algorithms to each subaperture, or a directional wavenumber spectrum spectrum parameterized by the depth of each subaperture. For this we consider each subaperture to have 64 elements, or an approximate resolution of 2 degrees. The apertures are overlapped by 32 elements, so each step in depth corresponds to an increment of 48 meters.

\section{RESULTS}

Figure 2 illustrates two of these depth dependent spectra for ranges of $120 \mathrm{~km}$, or two convergence zones (CZ's), and 150 km, or two and one half CZ's. We label such figures as a depth-direction sine (3)

${ }^{1}$ Both the internal wave and RAM MATLAB codes were provided by Matt Dzieciuh of Scripps Institute of Oceanography.

${ }^{2}$ A spacing of a $1 / 4$ wavelength would suffice because high angle wave components were suppressed by an absorbing bottom boundary. 
spectrum. (While CZ propagation is usually discussed in the context of shallow sources and receivers, the RAM solution reflects a CZ like behavior.) The figures have encapsulate the ray arrivals very
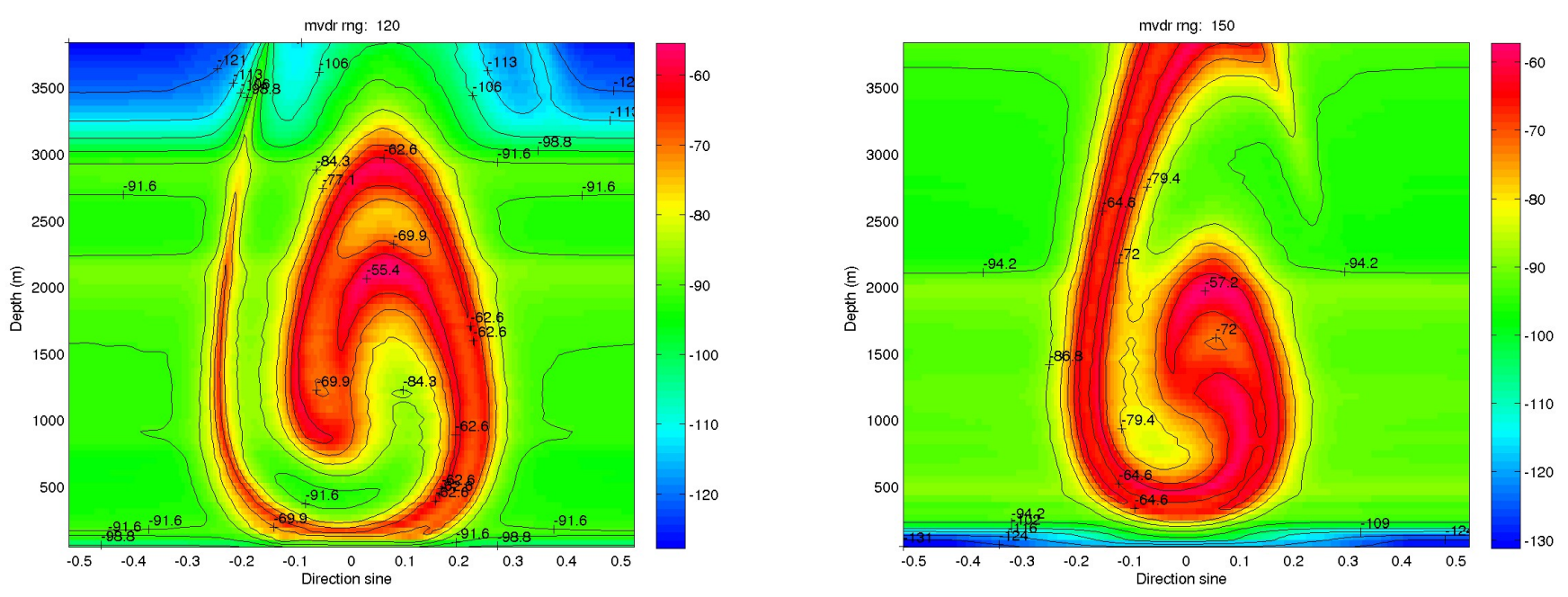

Figure 2: Left: Directional wave spectra vs subaperture depth at $120 \mathrm{~km}$; Right: Directional wave spectra vs subaperture depth at $150 \mathrm{~km}$. Note the subaperture depth is on the vertical and direction sine on the horizontal and is measured normal to the VLA, or the usual grazing angle.

concisely resembling what is often termed and angle-depth diagram. At the $120 \mathrm{~km}$ range we can identify two paths at the shallow subapertures corresponding to signals refracted near the sea surface. As we progress deeper there are four paths corresponding to two additional signals for the signals ducted along the sound channel axis at $1000 \mathrm{~m}$ where they were launched at the source depth. Going deeper near $1500 \mathrm{~m}$ we can identify four paths up to $2000 \mathrm{~km}$. Finally we loose the high angle paths and observe just those signals turning at low grazing angles. The depth dependent spectra for $150 \mathrm{~km}$, or between a CZ range, has a quite different structure. First, there are no shallow rays near the surface. Significant energy does not appear until approximately $250 \mathrm{~m}$. After this there are just two paths until $1500 \mathrm{~m}$. One is a high angle diving path with a negative D/E, while the other is a packet of positive D/E angles spread from grazing to nearly a direction sine of .2, or approximately 10 degrees. After this there is but one signal with a negative $\mathrm{D} / \mathrm{E}$ until the critical depth of the profile when two paths reappear. A very interesting analysis can be done while observing the stepping out in range as the structure of the spectra changes. Space does not allow including such a figure. Eventually, we expect that the propagation will "homogenize" with a mostly symmetric depth-direction sine spectrum. Eventually, bottom interaction ceases to absorb energy. Then, the volume scattering introduced by the internal wave perturbations then will lead to an "equipartition" of all the propagating rays and/or modes. At these ranges there is no potential to exploit the vertical structure. One way of quantifying the coherence of the signals both at each subaperture and the among them involves using the covariance matrices for the array data at eace subaperture. Two measures using thess are the mutual information and the cross coherence among the subapertures. There is in fact a one to one relationship between the two measures. The mutual information is a quantitative measure of how much information one signal vector provides about another. It is given by

$$
I\left(\mathbf{X}_{1}, \mathbf{X}_{2}\right)=-E\left[p_{\mathbf{x}_{1}, \mathbf{x}_{2}}\left(\mathbf{X}_{1}, \mathbf{X}_{2}\right)\right]
$$


For complex Gaussian random vectors this is given by

$$
I\left(\mathbf{X}_{1}, \mathbf{X}_{2}\right)=\log _{2}\left[\frac{\left|\Sigma_{\mathbf{x}_{1}, \mathbf{x}_{1}}\right|\left|\Sigma_{\mathbf{x}_{2}, \mathbf{x}_{2}}\right|}{\left|\Sigma_{\mathbf{x}_{1}, \mathbf{x}_{2}}\right|}\right]
$$

, where $\Sigma$ denotes the auto-covariances and cross-covariances according to the subscripts and the $|\cdot|$ is the determinant operator. By using the properties of partitions of determinants and their combination leads to an expression of the mutual information in terms of the magnitude of the coherence metric, or

$$
I\left(\mathbf{X}_{1}, \mathbf{X}_{2}\right)=-\log _{2}\left[1-\left|\rho\left(\mathbf{X}_{1}, \mathbf{X}_{2}\right)\right|^{2}\right]
$$

with

$$
\left|\rho\left(\mathbf{X}_{1}, \mathbf{X}_{2}\right)\right|^{2}=\frac{\left|\Sigma_{\mathbf{x}_{1}, \mathbf{x}_{2}}\right|}{\left|\Sigma_{\mathbf{x}_{1}, \mathbf{x}_{1}}\right|\left|\Sigma_{\mathbf{x}_{2}, \mathbf{x}_{2}}\right|}
$$

which is the multivariate definition of coherence.

We have applied this to covariances computed by using the ensemble of the PE realizations on the subapertures. ${ }^{3}$ Another measure of coherence is the number of degrees of freedom (dofs)for an ensemble of signal vectors observed on an a subaperture as well as for a pair of subapertures. For a single subaperture a measure of this is given in terms of the eigenvalues of a covariance as well,(5)

$$
\operatorname{dofs}=\frac{\left.\left[\sum_{i}^{N} \lambda_{i}\right)\right]^{2}}{\sum_{i}^{N} \lambda_{i}^{2}}=\frac{[\operatorname{Trace}(\Sigma)]^{2}}{\operatorname{Trace}\left(\Sigma^{2}\right)}
$$

where the $\lambda_{i}$ are the eigenvalues of the matrix $\Sigma$.

We are applying all these measure to assess the wavefield coherence. In the near future we plan to implement the two measures described. After we understand the issues using the simulated data, especially the statistical and calibration ones, we plan to applying this to the DVLA data of the Phil Sea.

\section{IMPACT/APPLICATIONS}

There are a number of ongoing plans for the use of large vertical apertures for Navy systems. Some of these are at the low frequencies used in the Phil Sea experiments, while other are at mid frequencies of a few kHz. The aperture scales mostly scales as the inverse of the frequency; nevertheless, the coherence of the multipaths is an important factor in the design of signal processing for VLA's. All current algorithms assume incoherent paths, similar to the distribution in the horizontal originating from uncorrelated sources. An important implication is that there may be less SGD and higher NG once we understand the statistics of the multipath ooherences.

\footnotetext{
${ }^{3}$ There is a significant statistical issue for computing these covariances beyond the discussion here. It is often suggested that one needs sample support on the order of $3 \mathrm{X}$ the number of sensors which implies approximately 200 realizations for statistical stability of the low order eigenvalues. This is very much an issue for a determinant since it is given by the product of the eigenvalues.
} 


\section{REFERENCES}

[1] Baggeroer, A.B., Kuperman, W.A., and Mikhalevsky, P.N.,'An overview of matched field processing methods in ocean acoustics", IEEE Journal on Oceanic Engineering, vol 18(4), pp. 401 - 424, [October 1993]

[2] Baggeroer, A.B., "Why dida applications of MFP fail, or did we not understand how to apply MFP", Proceedings of 1st Internatonal Conference and Exhibition on Underwater Acoustics, Kos, Greece, [June 2013]

[3] Dr. Matt Dzieciuch, personal communication

[4] Worcester, P.F., Andrew, R.K., Baggeroer, A.B., Colosi, J.A., D’Spain, G., Dzieciuch, M.A., Heaney, K.D., Howe, B.M., Kemp, J.N. and Mercer, J.A.,'Acoustic propagation and ambient noise in the Philippine Sea: the 2009 and 2010-2011 Philippine Sea experiments",Journal of the Acoustical Society of America vol 128, pp. 2385 [2010]

[5] Brillinger, D.R.,Time Series: Data Analysis and Theory, Holt Reinhart and Winston, International Series in Decision Processes, New York [1975]

\section{RELATED PROJECTS}

1. Vector Sensor Array Signal Processing N00014-07-12-0050

2. WHOI MURI on acoustic communications

3. SECNAV/CNO Chair "white paper" on recommendations to the CNR for an ONR Arctic acoustics program

4. SSTAG (Submarine Surveillance Technical Advisory Group) for N975)

5. FCP (Future Concepts Program for Design for Undersea Warfare) for COMSUBFOR) 